TRANSACTIONS OF THE

AMERICAN MATHEMATICAL SOCIETY

Volume 349, Number 4, April 1997, Pages 1359-1376

S 0002-9947(97)01869-2

\title{
RANDOMNESS AND SEMIGENERICITY
}

\author{
JOHN T. BALDWIN AND SAHARON SHELAH
}

\begin{abstract}
Let $L$ contain only the equality symbol and let $L^{+}$be an arbitrary finite symmetric relational language containing $L$. Suppose probabilities are defined on finite $L^{+}$structures with 'edge probability' $n^{-\alpha}$. By $T^{\alpha}$, the almost sure theory of random $L^{+}$-structures we mean the collection of $L^{+}$-sentences which have limit probability 1. $T_{\alpha}$ denotes the theory of the generic structures for $\mathbf{K}_{\alpha}$ (the collection of finite graphs $G$ with $\delta_{\alpha}(G)=|G|-\alpha \cdot \mid$ edges of $G \mid$ hereditarily nonnegative).

0.1. Theorem. $T^{\alpha}$, the almost sure theory of random $L^{+}$-structures, is the same as the theory $T_{\alpha}$ of the $\mathbf{K}_{\alpha}$-generic model. This theory is complete, stable, and nearly model complete. Moreover, it has the finite model property and has only infinite models so is not finitely axiomatizable.
\end{abstract}

This paper unites two apparently disparate lines of research. In [8], Shelah and Spencer proved a 0-1-law for first order sentences about random graphs with edge probability $n^{-\alpha}$ where $\alpha$ is an irrational number between 0 and 1. Answering a question raised by Lynch [5], we extend this result from graphs to hypergraphs (i.e. to arbitrary finite symmetric relational languages). Let $T^{\alpha}$ denote the set of sentences with limit probability 1 . The Spencer-Shelah proof proceeded by a process of quantifier elimination which implicitly showed the theories $T^{\alpha}$ were nearly model complete (see below) and complete.

Hrushovski in [3] refuted a conjecture of Lachlan by constructing an $\aleph_{0}$-categorical strictly stable pseudoplane. Baldwin and Shi [1] considered a variant on his methods to construct strictly stable (but not $\aleph_{0}$-categorical) theories $T_{\alpha}$ indexed by irrational $\alpha$. In this paper we show that for each irrational $\alpha, T^{\alpha}=T_{\alpha}$ and thus deduce that $T_{\alpha}$ is not finitely axiomatizable and that $T^{\alpha}$ is stable.

Each $T_{\alpha}$ is the theory of a 'generic' model $M_{\alpha}$ of an amalgamation class $\mathbf{K}_{\alpha}$ of finite structures. Although the Hrushovski examples are easily seen to be nearly model complete this is less clear for the $T_{\alpha}$ since they are not $\aleph_{0}$-categorical. We show that each $T_{\alpha}$ is nearly model complete.

In the first, purely model theoretic, section of the paper we describe our basic framework and prove a sufficient condition for certain theories, including the $T_{\alpha}$, to be nearly model complete. These conditions depend upon a generalization of the notion of genericity of a structure: semigenericity, which is introduced in this paper. In the second section we consider the addition of random relations and

Received by the editors September 7, 1994.

1991 Mathematics Subject Classification. Primary 03C10, 05C80.

Key words and phrases. Random graphs, 0-1-laws, stability.

Partially supported by NSF grant 9308768 and a visit to Simon Fraser University.

This is paper 528. Both authors thank Rutgers University and the Binational Science Foundation for partial support of this research.

(C)1997 American Mathematical Society 
deduce the main results for this case: The almost-sure theory and the theory of the generic model are equal, complete, stable, nearly model complete, and not finitely axiomatizable. From the model theoretic standpoint the extension from graphs to an arbitrary finite relational language is not a big step; it was spelled out in [9]. The distance is larger from the probability standpoint and the problem of making such an extension had been raised by Lynch [5].

The first author greatly benefited from discussions on this paper with M. Albert, G. Cherlin, M. Itai, A.H. Lachlan, C. Laskowski, D. Kueker and D. Marker. We want to thank Shmuel Lifsches for a careful reading of Section 1.

\section{NeAR MODEL COMPLETENESS}

After Hrushovski's construction of counterexamples to the conjectures of Lachlan and Zil'ber a number of authors explored generalizations of the variation he had introduced on the Fraissé-Jonsson construction. Hrushovski had noted that in his situation, where the generic model was $\omega$-saturated, the theory of the generic admitted the level of quantifier elimination which we christen 'nearly model complete' in this paper. We reprise one general setting for this study here and in the next section connect it with certain random models. Baldwin and Shi [1] studied a situation where the homogeneous-universal model, renamed generic by Kueker and Laskowski [4], is not $\omega$-saturated. Kueker and Laskowski investigated the conditions in which the theory constructed from a generic admitted various levels of quantifier elimination. After the first author noticed the connection between [3] and [8], we began to consider the quantifier complexity of the theory $T^{\alpha}$. There is no explicit elimination of quantifiers result in [8] but a lemma similar to our Lemma 1.30 is the crucial technical step. The second author had already begun notes generalizing [8]; the 0-1-law in Section 2 contains a more concrete version of his approach. Shelah has continued this approach to the probability aspect in more generality in [6]. A close look at the quantifier elimination results in $[3,8]$ suggests the following definition.

1.1. Definition. A theory $T$ is said to be nearly model complete if every formula is equivalent in $T$ to a Boolean combination of $\Sigma_{1}$-formulas.

Thus, $T$ is nearly model complete if the type of any finite sequence is determined by exactly the family of $\Sigma_{1}$-formulas it satisfies. Near model completeness lies strictly in strength between model completeness and 1-model completeness (every formula is equivalent to a $\Sigma_{2}$-formula).

1.2. Notation. Fix a finite relational language $L$. For any class $\mathbf{K}$ of structures, $S(\mathbf{K})$ denotes the class of all substructures of members of $\mathbf{K}$. Let $\mathbf{K}_{0}$ be a collection of finite $L$-structures and $\mathbf{K}$ be a class of models whose finite substructures are in $S\left(\mathbf{K}_{0}\right)$. We always assume that the empty structure is in $\mathbf{K}_{0}$. We will consider several different choices for $\mathbf{K}_{0}$ in this paper. In the following, $A, B, C$ vary over $\mathbf{K}_{0} ; M, N$ over $\mathbf{K}$. If $A, B$ are subsets of $N$, we write $A B$ for the $L$-structure contained in $N$ with universe $A \cup B$.

If $B \cap C=A$ we write $B \otimes_{A} C$ for the structure with universe $B \cup C$ and no relations other than those on $B$ or $C$. If $A, B, C$ are substructures of $N$ such that the structure imposed by $N$ on $B C$ is isomorphic to $B \otimes_{A} C$ we say $B$ and $C$ are freely joined over $A$ in $N$. In general we do not assume $\mathbf{K}_{0}$ is closed under $\otimes$ but this assertion will turn out to be an important property of some classes we consider. We write $X \subseteq_{\omega} Y$ to indicate $X$ is a finite subset of $Y$. 
We will first discuss a class of finite structures equipped with a dimension function satisfying certain natural properties. Then we define from this dimension function a notion of strong submodel. The main quantifier elimination result is proved in terms of the strong submodel concept. But, the connection with random models is obtained by exploiting an appropriate dimension function. The fact that this dimension function (in Example 1.6) is the same as that employed by Hrushovski to construct a strictly stable $\aleph_{0}$-categorical pseudoplane is the key to the argument for the stability of the almost sure theory of random graphs with edge probability $n^{-\alpha}$ when $\alpha$ is irrational.

1.3. Definition. Let $\delta$ be an arbitrary function assigning a real number to each isomorphism type of finite $L$-structure with $\delta(\emptyset)=0 . \delta(A / B)$ equals by definition $\delta(A B)-\delta(B)$. This yields immediately:

$$
\delta(A B / C)=\delta(A / B C)+\delta(B / C) .
$$

Note that the structure with universe $A B$ (and thus $\delta(A / B)$ ) is not determined by the separate structures on $A$ and $B$ but by some embedding of both into an element of $\mathbf{K}_{0}$.

1.4. Notation. We deal only with structures on which the relations of $L$ are symmetric (i.e $R(\bar{a})$ holds just if it holds for any permutation of $\bar{a}$ ) and irreflexive (i.e. hold only for sequences of distinct elements). Thus the relations are on sets rather than sequences.

We require the following conditions on $\delta$.

1.5. Axiom. $\mathbf{K}_{0}$ and $\delta$ satisfy for $A, B, C \ldots \in S\left(\mathbf{K}_{0}\right)$ and $N, M, \in \mathbf{K}$ :

i) $\delta: S\left(\mathbf{K}_{0}\right) \mapsto \Re^{+}$(the nonnegative reals) and $\delta(\emptyset)=0$.

ii) If $A, B$, and $C$ are disjoint subsets of $N$ then $\delta(A / B) \geq \delta(A / B C)$.

iii) For every $n \in \omega$ there is an $\epsilon_{n}>0$ such that if $|C|<n$ and $A, C$ are disjoint subsets of $M$ with $\delta(C A / A)<0$ then $\delta(C A / A) \leq-\epsilon_{n}$.

iv) There is a real number $\epsilon>0$ such that if $A, B, B^{\prime}$ are disjoint subsets of a model $N$ and $\delta(A / B)-\delta\left(A / B B^{\prime}\right)<\epsilon$ then $R\left(A, B, B^{\prime}\right)=\emptyset$ and $\delta(A / B)=$ $\delta\left(A / B B^{\prime}\right)$.

v) If $f$ is a 1-1 homomorphism from $A$ to $B$ then for every $X \subseteq Y \subseteq A, \delta(Y / X) \geq$ $\delta(f(Y) / f(X))$.

Axioms iii) and iv) play no explicit role in the argument presented here. But they are important in establishing the stability of $T_{\alpha}$ in [1] so are used in the proof of Theorem 1.34. Note that Axiom 1.5 iv) is stronger than the assertion that if $f$ is a 1-1 homomorphism, $\delta(X) \geq \delta(f(X))$.

Axiom $1.5 \mathrm{i})$ requires that the range of $\delta$ be the nonnegative reals. This allows us to obtain an important monotonicity property by modifying $\delta$ to $d: \mathbf{K} \times S\left(\mathbf{K}_{0}\right) \mapsto$ $\Re^{+}$by defining for each $N \in \mathbf{K}$,

$$
d(N, A)=\inf \left\{\delta(B): A \subseteq B \subseteq_{\omega} N\right\} .
$$

We usually write $d(N, A)$ as $d_{N}(A)$. We will omit the subscript $N$ if it is clear from context. This operator serves only as a notational convenience within this paper but plays an essential role in establishing the stability of $T_{\alpha}$ in [1]. The nonnegativity requirement on $\delta \mid \mathbf{K}_{0}$ not only justifies the definition of $d_{N}(A)$ but is necessary for the important Lemma 1.17. 
The classes $\left(\mathbf{K}_{\alpha}, \delta_{\alpha}\right)$, which are defined as follows, are important examples of this situation.

1.6. Example. Let the relation symbols of $L$ be $\left\langle R_{i}: i<p\right\rangle$. Let $w_{i}(A)$ be the number of realizations of $R_{i}$ in $A$. Fix a sequence $\bar{\alpha}$ with $0<\alpha_{i} \leq 1$ for $i<p$. Then for each $A$, let $e(A)=\sum w_{i} \alpha_{i}$. Let $\mathbf{K}_{\alpha}$ denote the class of all finite $L$-structures $A$ such that for all substructures $A^{\prime}$ of $A$,

$$
\delta_{\alpha}\left(A^{\prime}\right)=\left|A^{\prime}\right|-e\left(A^{\prime}\right) \geq 0 .
$$

See [1] for the straightforward verification of the axioms in this example.

From the dimension function we define certain special notions of submodel which make it easier to formulate our argument.

1.7. Definition. For finite $A, B$ contained in $N$, define the relative dimension of $A$ over $B, d_{N}(A / B)$, as $d_{N}(A / B)=d_{N}(A B)-d_{N}(B)$. If $B \subseteq A \subseteq \omega N$ this simplifies to $d_{N}(A / B)=d_{N}(A)-d_{N}(B)$

1.8. Definition. For $M \subseteq N \in S(\mathbf{K})$, define $M \leq_{s} N$ if for each finite $X \subseteq M$, $d_{M}(X)=d_{N}(X)$. We say $M$ is a strong submodel of $N$. We say $f: M \mapsto N$ is a strong embedding if $f M \leq_{s} N$. We write $M<_{s} N$ if $M \leq_{s} N$ but $M \neq N$.

We introduce a second kind of distinguished substructure by defining $\leq_{i}$ from $\leq_{s}$ as follows. Note that the definition yields that $A \leq_{i} A$.

1.9. Definition. For $A, B \in S\left(\mathbf{K}_{0}\right), A \leq_{i} B$ if $A \subseteq B$ but there is no $A^{\prime}$ with $A \subseteq A^{\prime}<_{s} B$. If $A \leq_{i} B$, we say $B$ is an intrinsic extension of $A$.

In terms of the dimension function $A \leq_{i} B$ means $A=B$ or $\delta(B / A)<0$ and $\delta(B / A)<\delta\left(B^{\prime} / A\right)$ for any intermediate $B^{\prime}$.

1.10. Lemma. Consider the situation described in Definition 1.3. If $\delta$ is a dimension function satisfying the properties of Axiom 1.5 and $\leq_{s}$ is defined as in Definition 1.8 then $\left(\mathbf{K}, \leq_{s}\right)$ satisfies the following conditions for $M, N, N^{\prime} \in S(\mathbf{K})$.

A1. $M \leq_{s} M$.

A2. If $M \leq_{s} N$ then $M \subseteq N$.

A3. If $M \leq_{s} N \leq_{s} N$ then $M \leq_{s} N^{\prime}$.

A4. If $M \leq_{s} N, N^{\prime} \subseteq N$ then $M \cap N^{\prime} \leq_{s} N^{\prime}$.

A5. $\leq_{i}$ is preserved under 1-1 homomorphism.

A6. For all $M \in S(\mathbf{K}), \emptyset \leq_{s} M$.

1.11. Remark. Note that $\mathbf{A} 4$ implies that if $M, N, N^{\prime} \in S(\mathbf{K})$ and $M \leq_{s} N$, $N^{\prime} \subseteq N$ and $M \subseteq N^{\prime}$ then $M \leq_{s} N^{\prime}$.

The quantifier elimination results of this section could be obtained by taking as primitive a class $\mathbf{K}_{0}$ equipped with a notion of strong submodel, and regarding the results of Lemma 1.10 and Lemma 1.17 below as axioms. Naturally, we would then require that $\mathbf{K}_{0}$ and $\leq_{s}$ be closed under isomorphism. The dimension function is needed for the calculations in Section 2.

1.12. Remark. In earlier formulations, the relation $\leq_{s}$ was defined just on $\mathbf{K}_{0}$ rather than on $S\left(\mathbf{K}_{0}\right)$. This leads to difficulties in phrasing Axiom A4. Our current formulation extends the ideas of [1] to encompass the Baudisch construction of a new $\aleph_{1}$-categorical group [2]. For our purposes in this paper, we could have identified $\mathbf{K}_{0}$ with $S\left(\mathbf{K}_{0}\right)$ and we make that restriction in Section 2. 
1.13. Remark. Axiom $\mathbf{A 6}$ holds in the examples at hand because the range of $\delta$ is nonnegative as specified in Axiom 1.5. In Section 2 we will begin with a $\delta$ mapping all finite $L$ - structures into the reals. The requirement that $\delta$ is nonnegative requires revising the choice of $\mathbf{K}_{0}$ (and thus $\mathbf{K}$ ) to guarantee that if $M \in \mathbf{K}$, then for every $A \subseteq \omega M, \delta(A) \geq 0$. We show it is harmless to make this assumption in Lemma 2.19.

1.14. Lemma. i) For any $A \subseteq C \in \mathbf{K}_{0}$, we can choose $B$ with $A \leq_{i} B \leq_{s} C$.

ii) $\leq_{i}$ is transitive.

Proof. For i), let $B$ have minimal cardinality among the subsets $X$ of $C$ that contain $A$ with $X \leq_{s} C$. Use $\mathbf{A} 4$ for ii).

1.15. Definition. For any $L$-structure $M$, let $A, B$ be finite substructures of $M$ with $A \subseteq B$. Then

1. By a copy of $B$ over $A$ in $M$ we mean the image of an extension $\hat{f}$ to $B$ of an embedding $f$ from $A$ into $M$.

2. $\chi_{M}(B / A)$ is the number of distinct copies of $B$ over $A$ in $M$.

3. $\chi_{M}^{*}(B / A)$ is the supremum of the cardinalities of maximal families of disjoint (over $A$ ) copies of $B$ over $A$ in $M$.

1.16. Lemma. If $\mathcal{B}$ and $\mathcal{B}^{\prime}$ are maximal families of disjoint over $A$ copies of $B$ over $A$ then $|\mathcal{B}| \leq|B-A|\left|\mathcal{B}^{\prime}\right|$.

Proof. Define a map from $\mathcal{B}$ to $\mathcal{B}^{\prime}$ by mapping each element of $\mathcal{B}$ to an element of $\mathcal{B}^{\prime}$ that it intersects off $A$. This map is at most $|B-A|$-to-one since the members of $\mathcal{B}$ are disjoint over $A$.

In particular, this shows that the supremum in the definition of $\chi_{M}^{*}$ is achieved. As one varies over the entire family of examples of structures constructed in this manner (e.g. in [3], [1], etc.) the dimension function produces an important trichotomy concerning pairs $A \subset B$. Consider an infinite $\left(\mathbf{K}_{0}, \leq_{s}\right)$-generic (Definition 1.23) model $M . \chi_{M}^{*}(B / A)$ will be bounded if $A \leq_{i} B$, infinite if $\delta(B / A)>0$, and will vary with the choice of $\left(\mathbf{K}_{0}, \leq_{s}\right)$ if $\delta(B / A)=0$. The key to the $0-1$ law in Section 2 is that when $\alpha$ denotes a sequence, which is linearly independent with 1 , the third case cannot occur. The uniform bound on $\chi_{M}^{*}(B / A)$ follows from our restricting $\mathbf{K}_{0}$ so $\delta$ is nonnegative. In [7], Shelah considers a different probability measure which does not permit the nonnegativity restriction; in that situation $\chi_{M}^{*}(B / A)$ is a slow growing function. In our situation we have the following.

1.17. Lemma. There is a binary function $t: \omega \times \omega \mapsto \omega$ which is monotone increasing in both arguments such that if $A \leq_{i} B$ then for any $M \in \mathbf{K}$ with $A \subseteq M$, $\chi_{M}(B / A) \leq t(|A|,|B|)$.

1.18. Remark. This follows easily from Lemma 3.19 of [1]. One must note that $A \leq_{i} B$ if and only if there is a sequence $A=A_{0}, A_{1}, \ldots, A_{n}=B$ such that $\left(A_{i}, A_{i+1}\right)$ is a minimal pair in the sense of [1].

1.19. Definition. For any $M \in \mathbf{K}$, any $m \in \omega$, and any $A \subseteq M$,

$$
\mathrm{cl}_{M}^{m}(A)=\bigcup\left\{B: A \leq_{i} B \subseteq M \&|B-A|<m\right\} .
$$

The following are immediate from Lemma 1.17 and the definitions.

1.20. Lemma. There is a function $f$ mapping $\omega \times \omega$ into $\omega$ such that for any $A \subseteq_{\omega} M \in \mathbf{K}$ and $m, \mathrm{cl}_{M}^{m}(A)$ is finite and its cardinality is uniformly bounded by $f(|A|, m)$. 
1.21. Lemma. For any $M, A, m, n$ there exists a $p$ depending on $|A|, m, n$ but not on the embedding of $A$ into $M$ with $\mathrm{cl}_{M}^{m}\left(\mathrm{cl}_{M}^{n}(A)\right) \subseteq \mathrm{cl}_{M}^{p}(A)$.

Proof. Let $p=m+f(|A|, n)$ and check.

The next result is immediate noting that $A \leq_{i} X$ does not depend on any ambient model containing $X$.

1.22. Lemma. If $A \subseteq B \subseteq C$ and $\mathrm{cl}_{C}^{m}(A) \subseteq B$, then $\mathrm{cl}_{C}^{m}(A)=\mathrm{cl}_{B}^{m}(A)$.

1.23. Definition. The countable model $M \in \mathbf{K}$ is $\left(\mathbf{K}_{0}, \leq_{s}\right)$-generic if

1. If $A \leq_{s} M, A \leq_{s} B \in \mathbf{K}_{0}$, then there exists $B^{\prime} \leq_{s} M$ with $B \cong_{A} B^{\prime}$; and

2. $M$ is the union of $\left\langle A_{i}: i<\omega\right\rangle$ where each $A_{i} \in \mathbf{K}_{0}$ and $A_{i} \leq_{s} A_{i+1}$.

1.24. Definition. A class $\left(\mathbf{K}, \leq_{s}\right)$ has the amalgamation property if for any three structures $A, B, C \in \mathbf{K}$ with strong embeddings $f, g$ from $A$ into $B, C$ there exist $D \in \mathbf{K}$ and strong embeddings $f^{\prime}: B \mapsto D, g^{\prime}: C \mapsto D$ with $f^{\prime} f=g^{\prime} g$.

Following the Fraissé-Jonsson construction, it is easy to show the following result.

1.25. Fact. If $\left(\mathbf{K}_{0}, \leq_{s}\right)$ satisfies $\mathbf{A} \mathbf{0}$ through $\mathbf{A 6}$ and has the amalgamation property then there is a unique countable $\left(\mathbf{K}_{0}, \leq_{s}\right)$-generic model.

We need a more local notion. This is the key new idea of this paper; it arose from the notion of a full model in [1] and from considering the role of $\mathrm{cl}_{M}^{m}(A)$ in [8].

1.26. Definition. The countable model $M$ is $\left(\mathbf{K}_{0}, \leq_{s}\right)$-semigeneric, or just semigeneric, if

1. $M \in \mathbf{K}$.

2. If $A \leq_{s} B \in \mathbf{K}_{0}$ and $g: A \mapsto M$, then for each finite $m$ there exists an embedding $\hat{g}$ of $B$ into $M$ which extends $g$ such that

(a) $\operatorname{cl}_{M}^{m}(\hat{g} B)=\hat{g} B \cup \mathrm{cl}^{m}(g A)$,

(b) $M \mid \mathrm{cl}_{M}^{m}(g A) \hat{g} B$ is the free join over $g A$ of $\mathrm{cl}_{M}^{m}(g A)$ and $\hat{g} B$.

In our applications any generic model is semigeneric (Lemma 1.35), so Fact 1.25 provides us with a semigeneric model. But while generic models are unique there are many semigeneric models in the situations that we deal with here.

We describe below an infinite set of first order formulas $\phi_{A, B, C}^{m}$ which allow us to axiomatize the class of semigeneric models by the following lemma, which is immediate once we have made the definitions. Note that these are $\Pi_{3}$-formulas as there is a universal quantifier hidden in the last clause.

1.27. Lemma. The structure $N \in \mathbf{K}$ is semigeneric, if and only if for each $A \leq_{s}$ $B$, each $m<\omega$, and $C \in \mathcal{D}_{A}^{m}, N \models \phi_{A, B, C}^{m}$

In establishing the following notation we are suppressing a fixed correspondence between enumerations of the structures $A, B, C, D$ and the variables $\bar{x}, \bar{z}, \bar{y}, \bar{w}$. This correspondence is chosen to preserve natural inclusions among the structures and the variables. Intuitively, $\mathcal{D}_{A}^{m}$ is the set of possible isomorphism types for $\mathrm{cl}_{M}^{m}(A)$. The structure $M$ satisfies $\phi_{A, B, C}^{m}$ just if the definition of semigenericity holds for the finite structures $A \subseteq C$ and $A \leq_{s} B$ when $C \approx \mathrm{cl}_{M}^{m}(A)$.

1.28. Notation. 1 . Write $A \leq_{i}^{m} D$ if for each $d \in D-A$, there is a $B$ with $A d \subseteq B, A \leq_{i} B$, and $|B-A|<m$. 
2. Let $A \in \mathbf{K}_{0}$.

$$
\mathcal{D}_{A}^{m}=\left\{D \in \mathbf{K}_{0}: A \leq_{i}^{m} D\right\} .
$$

Note that by Lemma 1.20 if $D \in \mathcal{D}_{A}^{m},|D|<f(|A|, m)$.

3. For $C \in \mathcal{D}_{A}^{m}$ let $\mathcal{D}_{A, C}^{m}$ be the set of $D \in \mathcal{D}_{A}^{m}$ which cannot be embedded in $C$.

4. For any finite $A, \delta_{A}(\bar{x})$ denotes the atomic diagram of $A$.

5. For $C \in \mathcal{D}_{A}^{m}, \theta_{A, C}^{m}(\bar{x}, \bar{y})$ is the formula

$$
\delta_{A}(\bar{x}) \wedge \delta_{C}(\bar{x}, \bar{y}) \wedge\left(\forall \bar{w}_{D}\right) \bigwedge_{D \in \mathcal{D}_{A, C}^{m}} \neg \delta_{D}\left(\bar{x}, \bar{w}_{D}\right) .
$$

Then for $\overline{a c}$ an enumeration of $C, A \subseteq C \subseteq N, N \models \theta_{A, C}^{m}(\bar{a}, \bar{c})$ if and only if $C=\operatorname{cl}_{N}^{m}(A)$.

6. For $A \leq_{s} B$ and $C \in \mathcal{D}_{A}^{m}$, let $\phi_{A, B, C}^{m}$ be the sentence

$$
(\forall \bar{x})(\forall \bar{y})(\exists \bar{z})\left[\delta_{A}(\bar{x}) \wedge \theta_{A, C}^{m}(\bar{x}, \bar{y}) \rightarrow\left(\delta_{C \otimes_{A} B}(\bar{x}, \bar{y}, \bar{z}) \wedge \theta_{B, C \otimes_{A} B}^{m}(\bar{x}, \bar{y}, \bar{z})\right)\right] .
$$

1.29. Theorem. If $\left(\mathbf{K}_{0}, \leq_{s}\right)$ satisfies $\mathbf{A} \mathbf{1}-\mathbf{A} \mathbf{6}$ of Lemma 1.10 and Lemma 1.17, then for every formula $\phi(\bar{x})$ there is a Boolean combination of existential formulas $\psi_{\phi}(\bar{x})$ such that if $M$ is $\left(\mathbf{K}_{0}, \leq_{s}\right)$-semigeneric then $\psi_{\phi}(\bar{x})$ is equivalent to $\phi(\bar{x})$ on $M$.

Proof. We first show:

1.30. Lemma. For any formula $\phi\left(x_{1} \ldots x_{r}\right)$ there is an integer $\ell=\ell_{\phi}$, such that for any pair of semigenerics $M, M^{\prime} \in \mathbf{K}$ and any $r$-tuples $\bar{a} \in M$ and $\bar{a}^{\prime} \in M^{\prime}$ if $\mathrm{cl}_{M}^{\ell_{\phi}}(\bar{a}) \approx \mathrm{cl}_{M^{\prime}}^{\ell_{\phi}}\left(\bar{a}^{\prime}\right)$ by an isomorphism taking $\bar{a}$ to $\bar{a}^{\prime}$, then $M \models \phi(\bar{a})$ if and only if $M^{\prime} \models \phi\left(\bar{a}^{\prime}\right)$.

Proof. The proof is by induction on formula complexity; the cases involving Boolean connectives are easy. So suppose $\phi(\bar{x})$ is of the form $(\exists y) \psi(\bar{x}, y)$. Suppose $M \models$ $\phi(\bar{a})$, so there is a $b$ such that $M \models \psi(\bar{a}, b)$.

Choose $p_{1}$ large enough so that for any $N \in \mathbf{K}$, any $r$-tuple $\bar{c}$ from $N$ and any $d \in N,\left|\operatorname{cl}_{N}^{\ell_{\psi}}(\bar{c}, d)\right|<p_{1}$. Set $p=\max \left(p_{1}, \ell_{\psi}\right)$. For $i \leq p$, for any $N \in \mathbf{K}$, for any $\bar{a} \in N$ define by induction $A_{0}^{N}=A_{0}^{N}(\bar{a})=\bar{a}$ and $A_{i+1}^{N}=A_{i+1}^{N}(\bar{a})=\operatorname{cl}_{N}^{p}\left(A_{i}\right)$. Now applying Lemma 1.21 , choose $\ell_{\phi}$ so that for every $\bar{a}$ of length $r$, and every semigeneric $N, A_{p}^{N}(\bar{a}) \subseteq \operatorname{cl}_{N}^{\ell_{\phi}}(\bar{a})$.

We want to show that for any semigenerics $M$ and $M^{\prime}$, for any $\bar{a} \in M^{r}, \bar{a}^{\prime} \in M^{\prime r}$, and $b \in M$ if $\operatorname{cl}_{M}^{\ell_{\phi}}(\bar{a}) \approx \operatorname{cl}_{M^{\prime}}^{\ell_{\phi}}\left(\bar{a}^{\prime}\right)$ then there is a $b^{\prime} \in M^{\prime}$ with $\operatorname{cl}_{M}^{\ell_{\psi}}(\bar{a}, b) \approx \operatorname{cl}_{M^{\prime}}^{\ell_{\psi}}\left(\bar{a}^{\prime}, b^{\prime}\right)$ by an isomorphism taking $\bar{a}$ to $\bar{a}^{\prime}$. Let $H_{0}$ be the substructure of $M$ with universe $(\bar{a}, b)$ and $H_{1}=\mathrm{cl}_{M}^{\ell_{\psi}}\left(H_{0}\right)$.

Fix $g$ which maps $\bar{a}$ to $\bar{a}^{\prime}$ and $\operatorname{cl}_{M}^{\ell_{\phi}}(\bar{a})$ isomorphically onto $\mathrm{cl}_{M}^{\ell_{\phi}}\left(\bar{a}^{\prime}\right)$. By the choice of $\ell_{\phi}$, for each $i \leq p, g$ maps $A_{i}^{M}(\bar{a})$ isomorphically onto $A_{i}^{M^{\prime}}\left(\bar{a}^{\prime}\right)$. (Use Lemma 1.22 and induct.) To avoid superscripts, for each $i$, let $A_{i}^{\prime}$ denote the image of $A_{i}=A_{i}^{M}$ under $g$. Notice that for some $j \leq p$,

$$
\left(A_{j+1}^{M}-A_{j}^{M}\right) \cap\left(H_{1}-H_{0}\right)=\emptyset .
$$

Since $p>\left|H_{1}-A_{j}^{M}\right|$ this implies $A_{j}^{M} \leq_{s} A_{j}^{M} H_{1}$.

Since $M^{\prime}$ is semigeneric, $M^{\prime} \models \phi_{A_{j}, H_{1}, A_{j+1}}^{p}$. Thus, there is an isomorphism $\hat{g}$ extending $g$ and mapping $H_{1}$ into $M$ with $\mathrm{cl}_{M^{\prime}}^{p}\left(A_{j}^{\prime} \hat{g} H_{1}\right)=\mathrm{cl}_{M^{\prime}}^{p}\left(A_{j}^{\prime}\right) \cup \hat{g} H_{1}$ and so that $M^{\prime} \mid\left(\mathrm{cl}_{M^{\prime}}^{p}\left(A_{j}^{\prime}\right) \hat{g} H_{1}\right)$ is a free join of $\mathrm{cl}_{M^{\prime}}^{p}\left(A_{j}^{\prime}\right)$ and $\hat{g} H_{1}$ over $A_{j}^{\prime}$. Let $H_{1}^{\prime}=\hat{g} H_{1}$ and $b^{\prime}=\hat{g}(b)$. We need to show $\operatorname{cl}_{M}^{\ell_{\psi}}(\bar{a}, b) \approx \operatorname{cl}_{M^{\prime}}^{\ell_{\psi}}\left(\bar{a}^{\prime}, b^{\prime}\right)$. 
By the choice of $\hat{g}$ and $H_{1}^{\prime}, A_{j}^{\prime} H_{1}^{\prime} \cong A_{j} H_{1}$ which contains $\mathrm{cl}_{M}^{\ell_{\psi}}(\bar{a}, b)$, so it suffices (by Lemma 1.22) to show $A_{j}^{\prime} H_{1}^{\prime}$ contains $\operatorname{cl}_{M^{\prime}}^{\ell_{\psi}}\left(\bar{a}^{\prime}, b^{\prime}\right)$. Note $\operatorname{cl}_{M^{\prime}}^{\ell_{\psi}}\left(\bar{a}^{\prime}, b^{\prime}\right) \subseteq$ $\mathrm{cl}_{M^{\prime}}^{p}\left(\bar{a}^{\prime}, b^{\prime}\right) \subseteq \operatorname{cl}_{M^{\prime}}^{p}\left(A_{j}^{\prime} \hat{g} H_{1}\right)=A_{j+1}^{\prime} H_{1}^{\prime}$. By Lemma 1.22, we have $\operatorname{cl}_{m^{\prime}}^{\ell_{\psi}}\left(\bar{a}^{\prime}, b^{\prime}\right)=$ $\mathrm{cl}_{A_{j+1}^{\prime} H_{1}^{\prime}}^{\ell_{\psi}}\left(\bar{a}^{\prime}, b^{\prime}\right)$. Since $A_{j+1}^{\prime}$ and $H_{1}^{\prime}$ are freely joined over $A_{j}^{\prime}, \hat{g}^{-1}$ is a $1-1$ homomorphism from $A_{j+1}^{\prime} H_{1}^{\prime}$ onto $A_{j+1} H_{1}$. Applying A5 from Lemma 1.10, we see $\mathrm{cl}_{A_{j+1}^{\prime} H_{1}^{\prime}}^{\ell_{i}}\left(\bar{a}^{\prime}, b^{\prime}\right) \subseteq A_{j}^{\prime} H_{1}^{\prime}$ whence $\mathrm{cl}_{A_{j+1}^{\ell_{\psi}} H_{1}^{\prime}}\left(\bar{a}^{\prime}, b^{\prime}\right)=\mathrm{cl}_{A_{j}^{\prime} H_{1}^{\prime}}^{\ell_{\psi}}\left(\bar{a}^{\prime}, b^{\prime}\right)$.

The proof of the following corollary encompasses the derivation of Theorem 1.29 from Lemma 1.30.

1.31. Corollary. Suppose there is a $\left(\mathbf{K}_{0}, \leq_{s}\right)$-semigeneric L-structure. The theory of the class of $\left(\mathbf{K}_{0}, \leq_{s}\right)$-semigeneric $L$-structures is nearly model complete.

Proof. We have shown that in each semigeneric model the truth of $\phi(\bar{a})$ is determined by the isomorphism type of $\mathrm{cl}_{M}^{\ell_{\phi}}(\bar{a})$ and does not depend on the particular embedding of $\mathrm{cl}_{M}^{\ell_{\phi}}(\bar{a})$ in $M$. There are only finitely many possibilities for this closure and each is determined by a conjunction of existential and universal sentences (specifying which $B$ with $|B|<\ell_{\phi}$ and with $\bar{a}$ enumerating an intrinsic substructure of $B$ occur).

1.32. Corollary. The theory of the semigeneric models is complete.

Proof. If $N$ is semigeneric, $N \in \mathbf{K}$ so, by A6, $N$ does not contain any substructure $A$, with $\delta(A)<0$. Thus, $\operatorname{cl}_{N}(\emptyset)=\emptyset$; completeness follows from Lemma 1.30.

Recall from [1]:

1.33. Definition. $\mathbf{K}_{0}$ has the full amalgamation property if $B \cap C=A$ and $A \leq_{s} B$ implies $D=B \otimes_{A} C \in \mathbf{K}_{0}$ and $C \leq_{s} D$.

The following result is proved in [1]

1.34. Theorem. $\left(\mathbf{K}_{\alpha}, \leq_{s}\right)$ has the full amalgamation property. There is a generic model $M_{\alpha}$ and the theory $T_{\alpha}$ of this generic model is stable.

Using the full amalgamation property, it is easy to see

1.35. Lemma. The generic model $M_{\alpha}$ for $\mathbf{K}_{\alpha}$ is semigeneric.

Combining the above results we have

1.36. Theorem. $T_{\alpha}$ is nearly model complete.

The strength of this remark is emphasized by the following observation.

1.37. Theorem. The theory $T_{\alpha}$ is not model complete.

Proof. If $T$ is model complete with generic $M$, the type of any finite subset $X$ is determined by positive assertions of the substructures that contain $X$. Fix $A<_{i}$ $B<_{i} C \in \mathbf{K}_{0}$. Suppose $A_{1}, A_{2} \subset M$ with $f: A \approx A_{1}$ and $f: A \approx A_{2}$ and suppose $A_{1}<_{i} B_{1} \leq_{s} M, A_{2}<_{i} C_{2} \leq_{s} M$ with $f$ and $g$ extending to $\hat{f}, \hat{g}$ such that $\hat{g}: B \approx B_{1}$ and $\hat{g}: C \approx C_{2}$. Then every existential formula true of $A_{1}$ is true of $A_{2}$ but the converse is obviously false. To see the nonobvious assertion, let $D$ be arbitrary with $A_{1} \subseteq D \subset M$ and $D$ not contained in $B_{1}$. Then, $B_{1} \leq{ }_{s} B_{1} D$. Pulling back to $A, B, C$, by full amalgamation, there is a $D^{\prime} \supset D$ such that $B D^{\prime} \otimes_{B} C \in \mathbf{K}_{0}$ and $C \leq_{s} C D^{\prime}$. Extending $\hat{g}$ from $C$ to $C D^{\prime}$ provides the required witness. 
1.38. Remark. Let $L$ contain a single binary relation and restrict to the class of graphs. Baldwin and Shi noted [1] that full amalgamation holds for the class $\mathbf{K}_{\alpha}^{\prime}$ consisting of those graphs in $\mathbf{K}_{\alpha}$ which omit squares. Laskowski observed that this argument applies as well to the class $K_{\alpha}^{n}$ of graphs which omit cliques of size $n$. Thus the theory of the generic model associated with each of these classes is stable and nearly model complete.

\section{AdDing RANDOM RELATions}

In this section we begin with the collection of finite models for a language $L$ with only the equality symbol (i.e. $n$-element sets for arbitrary $n$ ) and add additional 'random' relations with respect to probability measures described below.

We show that a 0-1-law holds for the set of first order sentences in the expanded language and that the almost sure theory (the sentences with limit probability 1) is stable. Adding a single symmetric irreflexive binary relation gives the family of theories investigated independently by Shelah-Spencer and Baldwin-Shi. Viewing this situation as an expansion of the language of equality may seem eccentric but we expect to exploit this viewpoint for more interesting base languages in the future. This project is well-advanced in [6].

2.1. Context. Let $L$ contain only the equality symbol. The $L$-structure $M_{n}$ is a set with $n$ elements. $\mathbf{K}_{0}$ is the class of all finite sets and $\mathbf{K}$ the class of all sets. On $\mathbf{K}, \leq_{s}$ is just $\subseteq$ and $A \leq_{i} B$ just if $A=B$.

2.2. Remark. The properties $\mathbf{A 1}-\mathbf{A 6}$ and the conclusion of Lemma 1.17 hold for $\mathbf{K}$ in Context 2.1. Moreover, $\mathbf{K}_{0}$ has the full amalgamation property.

2.3. Definition. We say that $B$ is a primitive extension of $A$ if $A \leq_{s} B$ and for every $B^{\prime}$ with $B^{\prime}$ properly contained between $A$ and $B, B^{\prime}$ is not a strong submodel of $B$.

Now, we show how to define the notion of independent random relations (with edge probability ' $n^{-\alpha}$ ') for an arbitrary finite relational language $L^{+}$. Then we define the notions of dimension and strong submodel in the extended language $L^{+}$ and show that the properties A1- $\mathbf{A 5}$ hold for the extended language and $\mathbf{A 6}$ holds with probability 1 .

2.4. Notation. We write $[X]^{m}$ for the collection of $m$-element subsets of a set $X$. We will write either $C \in[X]^{m}$ or (surreptitiously fixing an enumeration of $C$ ) $\bar{c} \in[X]^{m}$ to indicate a member of this set.

2.5. Adding random relations. Fix an enumeration $\left\langle R_{i}: i\langle p\rangle\right.$ of the relation symbols in $L^{+}-L$ and let $k_{i}$ denote the arity of $R_{i}$. Let $L_{i}$ contain only $R_{i}$. Let $t$ denote the largest arity of the $R_{i}$. Fix also a sequence of numbers $\alpha_{i}$ with $0<\alpha_{i} \leq 1$ and $\gamma_{i}$ with $0 \leq \gamma_{i} \leq 1$ for $i<p$. (We will require later that the $\alpha_{i}$ and 1 be linearly independent over the rationals.)

We will define, for each isomorphism type of an $L^{+}$structure of size $n$, the probability of a random structure of size $n$ having that isomorphism type.

We assume that each new relation in the expanded structure is symmetric and irreflexive in the sense of Paragraph 1.4. Note that this formalism does not describe what one should mean by a random directed graph. 
Let $N$ be an $L^{+}$structure of cardinality $n$. Let $C$, enumerated as $\bar{c}$, be a subset of $N$ with size $k_{i}$. Let

$$
q_{i, n}\left(C \mid L_{i}\right)= \begin{cases}\gamma_{i} n^{-\alpha_{i}} & \text { if } N \models R_{i}(\bar{c}), \\ 1-\gamma_{i} n^{-\alpha_{i}} & \text { if } N \models \neg R_{i}(\bar{c}),\end{cases}
$$

and

$$
P_{n}(N)=\prod_{i<p} \prod\left\{q_{i, n}(C): C \in[N]^{k_{i}}\right\} .
$$

2.6. Remark. Let $w_{j}(N)=\left|\left\{C \in[N]^{k_{j}}: N \models R_{j}(\bar{c})\right\}\right|$ and $\bar{w}_{j}(N)=\left|\left\{C \in[N]^{k_{j}}: N \models \neg R_{j}(\bar{c})\right\}\right|$. Then

$$
P_{n}(N)=\prod_{j<p}\left(\gamma_{j} n^{-\alpha_{j}}\right)^{w_{j}(N)}\left(1-\gamma_{j} n^{-\alpha_{j}}\right)^{\bar{w}_{j}(N)} .
$$

If $L^{+}$has a single binary edge relation and the probability of a two element structure is $n^{-\alpha}$ when the points are related and $1-n^{-\alpha}$ if not, we return to the situation of [8].

Recall from Lemma 1.27 the sentences axiomatizing the class of semigeneric models. We want to show that the almost sure theory exists and is exactly the theory of the semigenerics. To this end, we will show

$$
\lim _{n \rightarrow \infty} P_{n}\left(\phi_{A, B, C}^{m}\right)=1
$$

for each $m, A, B, C$.

2.7. Notation. Henceforth, $A, B, \ldots M, N \ldots$ range over $L$-structures. $A^{+}, B^{+}$ etc. denote an expansion of $A$, respectively $B$ to $L^{+}$. We refer to the universe of $A^{+}$ or $A$ by either of these terms rather than the more accurate $\left|A^{+}\right|$or $|A|$ and reserve | | for cardinality. Thus $A^{+} \mid L=A$ and we use these notations interchangeably.

We now translate our probability asssignment into a class $(\mathbf{K}, \delta)$ as in Example 1.6.

2.8. Notation. Let $\mathbf{K}_{0}^{*}$ be the collection of all finite $L^{+}$structures.

1. For $A^{+} \in \mathbf{K}_{0}^{*}$, define $\delta\left(A^{+}\right)=\delta_{\alpha}\left(A^{+}\right)$as in Example 1.6, using only the relation symbols in $L^{+}-L$ and using the parameters $\alpha_{i}$ from Paragraph 2.5.

2. $e\left(B^{+} / A^{+}\right)$denotes $e\left(A^{+} B^{+}\right)-e\left(A^{+}\right)$.

3. $\mathbf{K}_{0}^{+}$denotes the collection of $A^{+} \in \mathbf{K}_{0}^{*}$ such that for each $A^{\prime} \subseteq A, \delta\left(A^{\prime}\right) \geq 0$.

4. $\gamma\left(A^{+}, B^{+}\right)=\prod_{i<p}\left\{\gamma_{i}:|C|=k_{i}, C \subseteq B, C \nsubseteq A \& B \models R_{i}(\bar{c})\right\}$.

2.9. Remark. The link between the function $\delta$ and the probabilistic situation is provided in Remark 2.18 where we show that the expectation of the existence of a copy over $A^{+}$of a structure $B^{+}$is determined by $\delta\left(B^{+} / A^{+}\right)$. Lemma 2.19 implies that replacing $\mathbf{K}_{0}^{*}$ by $\mathbf{K}_{0}^{+}$does not change the almost sure theory. But it does make the model theory conform with the framework of Section 1. The major calculations of this section were carried out in $K_{0}^{*}$ in [8].

2.10. Assumption (Irrationality Hypothesis). The coefficients $\alpha_{i}$ and 1 are linearly independent over the rationals. This generalizes for an arbitrary finite language the assumption in the case of random graphs with $p=n^{-\alpha}$ that $\alpha$ is irrational. This hypothesis easily implies that for any $L$-structures $A \leq_{s} B$ and expansions $A^{+} \subseteq B^{+}, \delta\left(B^{+} / A^{+}\right) \neq 0$. This is a key property (see Remark 2.27). 
We have the notions of $\leq_{s}$ and $\leq_{i}$ as a relation on members of $\mathbf{K}_{0}$. The definition of $\delta$ on $\mathbf{K}_{0}^{+}$induces corresponding relations on $\mathbf{K}_{0}^{+}$. Since we work directly with $\delta$ it is worthwhile recording the translation.

2.11. Definition. For $A^{+} \subseteq B^{+} \in \mathbf{K}_{0}^{+}$,

1. $A^{+} \leq_{s} B^{+}$if $\delta\left(B_{1}^{+} / A^{+}\right)>0$ for every $B_{1}^{+}$with $A^{+} \subset B_{1}^{+} \subseteq B^{+}$.

2. $A^{+} \leq_{i} B^{+}$if $\delta\left(B^{+} / B_{1}^{+}\right)<0$ for every $B_{1}^{+}$with $A^{+} \subseteq B_{1}^{+} \subset B^{+}$.

3. $B^{+}$is a primitive extension of $A^{+}$, if $\delta\left(B^{+} / A^{+}\right)>0$ and $\delta\left(B^{+} / A_{1}^{+}\right) \leq 0$ for each $A_{1}^{+}$with $A^{+} \subset A_{1}^{+} \subseteq B^{+}$.

Note that:

2.12. Remark. $\mathbf{K}_{0}^{+}$satisfies axioms A0-A6. We will be using the following monotonicity properties which follow formally as in Section 1.

2.13. Lemma. 1. If $A^{+} \leq_{i} B^{+}$and $A^{+} \subseteq B_{1}^{+} \subseteq B^{+}$then $B_{1}^{+} \leq_{i} B^{+}$.

2. If $A^{+} \leq_{s} B^{+}$and $A^{+} \subseteq B_{1}^{+} \subseteq B^{+}$then $A^{+} \leq_{s} B_{1}^{+}$.

2.14. Remark. The exact phrasing of the following notions is extremely delicate. We consider a fixed pair of finite $L^{+}$-structures, $A^{+} \leq_{s} B^{+}$. The $L$-structures $\left\langle M_{n}: n<\omega\right\rangle$ naturally form a chain so an embedding $f$ of $A$ into $M_{n}$ can naturally be regarded as a map of $A$ into $M_{m}$ for $m>n$. We are concerned with the properties of extensions of $f$. Thus, the immediately following definition of an $L^{+}$homomorphism extending $f$ is agnostic concerning the preservation of relations on A.

2.15. Definition. Let $A^{+} \subseteq B^{+}$. Let $f$ be a 1-1 map from $A$ into $M_{n}$, and let $G^{+}$be an $L^{+}$structure expanding $M_{n}$. Let $T$ denote the range of $f$.

1. We say an injective map $g: B^{+} \mapsto G^{+}$which extends $f$ is an $L^{+}$-homomorphism over $A$ if for any $L^{+}$-relation $R$, and any $\bar{b} \in B$ but not in $A, B^{+}=R(\bar{b})$ implies $G^{+}=R(g \bar{b})$.

2. For any $G^{+}$expanding $M_{n}$, and $W \subseteq M_{n}$ with $|W| \leq n$ we say

$$
N\left(f, A^{+}, B^{+}, W\right)=k \text { in } G^{+},
$$

if

$k=\mid\left\{g: B^{+} \mapsto W \subseteq M_{n}\right.$ is an $L^{+}$-homomorphism over $A$ and $\left.g \supseteq f\right\} \mid$.

If $W=M_{n}$ we omit it.

3. We say $G^{+}$is in the event $Y_{f}$, which depends on a constant $c_{1}$, if

$$
n^{\delta\left(B^{+} / A^{+}\right)}(\log n)^{-(v(B / A)+1)}<N\left(f, A^{+}, B^{+}\right)<c_{1} n^{\delta\left(B^{+} / A^{+}\right)} .
$$

4. Let $U$ denote the range of $f$. For each $S \subseteq M_{n}$ with $S \cap U=\emptyset$ and $|S|=$ $|B-A|$, fix (if possible) an $L$-isomorphism $g_{S}$ between $B$ and $U S$ which extends $f$. (Since $L$-isomorphism just means 1-1 map, such $S$ and $g_{S}$ exist whenever $n \geq|B|$.)

5. For each such $S$ with fixed $L$-isomorphism $g_{S}$ of $B$ into $M_{n}$, let $X_{f, S}$ be a random variable such that $X_{f, S}\left(G^{+}\right)$is $\begin{cases}1 & \text { if } g_{S} \text { maps } B \text { onto } U S \text { and is an } L^{+} \text {-homomorphism over } A \text { into } G^{+}, \\ 0 & \text { otherwise. }\end{cases}$

For $W \subseteq M_{n}$ with $|W| \leq n$, let

$$
X_{f, W}=\sum\left\{X_{f, S}: g_{S}: B \mapsto M_{n} \text { and } g_{S} \supseteq f \text { and } S \subseteq W\right\} .
$$


If $W=M_{n}$, we write $X_{f}$ for $X_{f, W}$.

2.16. Notation. $\quad 1$. For any property $P$ of structures, in particular a first order property, the assertion, 'for almost all sufficiently large $M, M \models P^{\prime}$ (abbreviated a.a.) means 'for every $\epsilon>0$ there is an $N$ such that if $n>N$, $P_{n}\left(\left\{G^{+} \mid L=M_{n}: G^{+}=P\right\}\right)>1-\epsilon^{\prime}$.

2. By an indicator random variable we mean one which takes values 0 or 1 and thus indicates a set.

3. We write $f \approx g$ if $f=O(g)$ and $g=O(f)$.

The next lemma expresses the key observation linking the probability with the dimension function $\delta$.

2.17. Lemma. For all sufficiently large $n$ and all $f: A \mapsto M_{n}$, and any $W \subseteq M_{n}$ with $|W| \leq n$, the expectation

$$
E\left(X_{f, W}\right) \approx|W|^{v(B / A)} n^{-e\left(B^{+} / A^{+}\right)} .
$$

Proof. The probability of an $L$-embedding of $B^{+} \mid L$ into $M^{+}$actually being an $L^{+}$homomorphism is $\gamma\left(A^{+}, B^{+}\right) n^{-e\left(B^{+} / A^{+}\right)}$. The number of such embeddings has order of magnitude $|W|^{v(B / A)}$. Since expectation is additive this yields

$$
E\left(X_{f, W}\right) \approx|W|^{v(B / A)} \gamma\left(A^{+}, B^{+}\right) n^{-e\left(B^{+} / A^{+}\right)} .
$$

The constant is absorbed by the approximation $\approx$. In particular, we have:

2.18. Remark. If $W=M_{n}$ this simplifies to

$$
E\left(X_{f}\right) \approx n^{\delta\left(B^{+} / A^{+}\right)} .
$$

In Theorem 2.30 we guarantee that our extensions are $L^{+}$-isomorphisms (no new relations) rather than just $L^{+}$-homomorphisms. Now we justify the restriction from $\mathbf{K}_{0}^{*}$ to $\mathbf{K}_{0}^{+}$.

2.19. Lemma. If $\delta\left(B^{+}\right)<0$ then a.a. there is no embedding of $B^{+}$into $G^{+}$.

Proof. The expected number of copies of $B^{+}$is $n^{\delta\left(B^{+}\right)}$. If $\delta\left(B^{+}\right)<0$, this tends to 0 .

2.20. Theorem. Fix $A^{+} \subseteq B^{+}$with $A^{+} \leq{ }_{s} B^{+}$.

Let $V$ be the event: for all $f: A \mapsto M_{n}$, the event $Y_{f}$ holds.

Then, for some choice of $c_{1}$ (recall $Y_{f}$ depends on $\left.c_{1}\right)$,

$$
\lim _{n \rightarrow \infty} P_{n}(V)=1 \text {. }
$$

Proof. By a straightforward induction, we can reduce to the case that $B^{+}$is a primitive extension of $A^{+}$. The proof of this case proceeds through several definitions and lemmas. Considering the definition of $Y_{f}$, one can see that we need to establish both lower and upper bounds. The lower bound argument proceeds as follows. Roughly speaking, for $f: A \mapsto M_{n}$ and $W \subseteq M_{n}$, we say $(f, W)$ is bad if there is no extension of $f$ to an $L^{+}$-homomorphism (in the sense of Definition 2.15) of $B^{+}$into $W$. In Lemma 2.22 we show that if $W$ meets a cardinality requirement specified in Definition 2.21 then the probability that $(f, W)$ is bad is less than $1 / 2$. By strengthening the requirements on $W$ as in Definition 2.23 we improve the upper bound on the probability that $(f, W)$ is bad in Lemma 2.24. Finally, taking into account the number of possible $W$ 's, we complete the proof of the lower bound in 
Paragraph 2.25. After several preliminary definitions and lemmas we complete the proof of the upper bound in Paragraph 2.29.

2.21. Definition. We have fixed $A^{+} \subseteq B^{+}$with $B^{+}$a primitive extension of $A^{+}$. For $G^{+}$an $L^{+}$-expansion of $M_{n}$ and $W \subseteq M_{n}$ and $f$ an $L$-isomorphism of $A$ into $M_{n},(f, W)$ is bad in $G^{+}$if there is no $g$ defined on $B-A$ into $W$ such that $f \cup g$ defines an $L^{+}$-homomorphism from $B^{+}$into $G^{+}$.

2.22. Lemma. There is a constant s such that for all sufficiently large $n$ and any $L$-isomorphism $f: A \mapsto M_{n}$, if $|W|$ is the integer $m=m_{s}=\left[s n^{e\left(B^{+} / A^{+}\right) / v(B / A)}\right]$ then

$$
P_{n}((f, W) \text { is bad })<1 / 2 .
$$

Proof. Without serious loss of precision, $W \cap \operatorname{rng} f=\emptyset$. We use the notation from Definition 2.15.

Now

$$
P_{n}((f, W) \text { bad })=P_{n}\left(X_{f, W}=0\right)
$$

so we want to show that for all sufficiently large $n$,

$$
P_{n}\left(X_{f, W}=0\right)<1 / 2 .
$$

By Chebyshev's inequality,

$$
P_{n}(X=0) \leq \frac{\operatorname{Var}(X)}{E(X)^{2}} .
$$

By Lemma 2.17,

$$
E(X)=E\left(X_{f, W}\right) \approx|W|^{v(B / A)} n^{-e\left(B^{+} / A^{+}\right)} .
$$

Using the fact that $|W|=m_{s}$ this shows $E(X)$ is a polynomial of degree $v$ in $s$, as the powers of $n$ cancel. We will obtain the required result by showing $\operatorname{Var}(X)$ is a polynomial of degree $2 v-1$ in $s$ which implies that for sufficiently large $s$, $\frac{\operatorname{Var}(X)}{E(X)^{2}}<1 / 2$.

Now,

$$
\operatorname{Var}(X)=\sum_{S} \operatorname{Var}\left(X_{S}\right)+\sum_{S \neq T} \operatorname{Cov}\left(X_{S}, X_{T}\right)
$$

where $S, T$ range over subsets of $M_{n}$ disjoint from the image of $f$.

An easy calculation shows that for any set of indicator random variables

$$
E\left(\sum_{S} X_{S}\right) \geq \sum_{S} \operatorname{Var}\left(X_{S}\right)
$$

so we have

$$
\operatorname{Var}(X) \leq E(X)+\sum_{j=0}^{v} \sum_{|(S \cap T)-A|=j} \operatorname{Cov}\left(X_{S}, X_{T}\right) .
$$

If $|(S \cap T)-A|=0$ then $S \cap T=\emptyset$ and $\operatorname{Cov}\left(X_{S}, X_{T}\right)$ is zero. Always, $\operatorname{Cov}\left(X_{S}, X_{T}\right) \leq E\left(X_{S} X_{T}\right)$ which, since these are indicator random variables, is just $P_{n}\left(X_{S} X_{T}\right)$. Recall the definition of the probability measure from Definition $2.5 ; t$ is largest arity in the language. 


$$
\begin{gathered}
P_{n}\left(X_{S} X_{T}\right)=\prod_{m \leq t} \prod_{A \in[S T]^{m}} q_{m}\left(\left(M \mid L^{\leq m}\right) \mid A\right) \\
\leq \prod_{m \leq t}\left(\frac{\prod_{A \in[S]^{m}} q_{m}\left(\left(M \mid L^{\leq m}\right) \mid A\right) \prod_{A \in[T]^{m}} q_{m}\left(\left(M \mid L^{\leq m}\right) \mid A\right)}{\prod_{A \in[S \cap T]^{m}} q_{m}\left(\left(M \mid L^{\leq m}\right) \mid A\right)} .\right.
\end{gathered}
$$

Let $B^{\prime}$ be $g_{s}^{-1}(S \cap T)$. So $\left|B^{\prime}\right|=j$. Abbreviating the notations from 2.8 , let $c_{S}=$ $c_{T}=\gamma\left(A^{+}, B^{+}\right)$and $c_{B^{\prime}}=\gamma\left(A^{+}, B^{\prime}\right)$. Similarly, let $e=e_{S}=e_{T}=e\left(B^{+} / A^{+}\right)$and $u^{\prime}=e\left(B^{\prime} / A^{+}\right)$. With this notation we can rewrite the last inequality as

$$
P_{n}\left(X_{S} X_{T}\right) \leq \frac{c_{S} c_{T}}{c_{B^{\prime}}} n^{-\left(2 e-u^{\prime}\right)} .
$$

(The key to the inequality is that $2 e-u^{\prime}$ may undercount the number of relations on $S T$ but this undercount can only overestimate the probability.) $|(S \cap T)-A|=j$, $|S T-A|=2 v-j$ so $\chi_{W}(S T / A) \approx m^{2 v-j}$.

If $\frac{u^{\prime}}{j}>\frac{e}{v}$ then $\frac{e}{v}>\frac{e-u^{\prime}}{v-j}$ which contradicts the fact that $B^{+}$is a primitive extension of $A^{+}$. So $u^{\prime} \leq \frac{j e}{v}$. Thus,

$$
\sum_{|(S \cap T)-A|=j} \operatorname{Cov}\left(X_{S}, X_{T}\right) \leq m^{2 v-j} n^{u^{\prime}-2 e} \leq\left(s n^{e / v}\right)^{2 v-j} n^{j e / v-2 e}=\left(s^{v}\right)^{2-j / v} .
$$

(We can drop the constants in the last computation as $\frac{c_{S} c_{T}}{c_{B}^{\prime}}<1$.) So,

$$
\operatorname{Var}(X) \leq E(X)+\sum_{j=1}^{v}\left(s^{v}\right)^{2-j / v} \leq E(X)+v s^{2 v-1} .
$$

Since $E(X)$ has degree $v$ in $s$, this implies $\operatorname{Var}(X) \leq E^{2}(X) / 2$ for sufficiently large $s$ and so

$$
P_{n}((f, W) \text { is bad })<1 / 2 .
$$

Now we want to modify the choice of $W$ to get a better upper bound on the probability that $(f, W)$ is bad.

2.23. Definition. Choose $s$ by Lemma 2.22. Again, let $m_{s}=\left[s n^{e\left(B^{+} / A^{+}\right) / v(B / A)}\right]$. We say that $W \subseteq M_{n}$ is $k$-appropriate if $|W|=\left[1+k m_{s} \ln n\right]$.

2.24. Lemma. For all sufficiently large $n$ and any L-isomorphism $f: A \mapsto M_{n}$, for sufficently large $k$, if $W \subseteq n$ is $k$-appropriate,

$$
P_{n}((f, W) \text { is bad })<\frac{n^{-|A|-1}}{2} .
$$

Proof. Again, assume without loss of generality that $W \cap A=\emptyset$. Suppose $W$ contains $k \ln n$ disjoint subsets $W_{i}$ each with cardinality $m_{s}$. For $(f, W)$ to be bad, each of the $k \ln n$ independent events that $\left(f, W_{i}\right)$ is bad must occur and by Lemma $2.22 P_{n}((f, W)$ is bad $)<1 / 2$. Thus,

$$
P_{n}((f, W) \text { is bad })<2^{-k \ln n} .
$$

But for all sufficiently large $n$ and $k$,

$$
2^{-k \ln n}<\frac{n^{-|A|-1}}{2}
$$

so we have the result. 
We have shown that for each $f$, a.a. there is a $W$ such that $(f, W)$ is not bad. The next paragraph strengthens this assertion.

2.25. Proof of Lower Bound in Theorem 2.20. Fix $k$ satisfying the conclusion of Lemma 2.24. For an $L$-isomorphism $f$ of $A$ into $M_{n}$, let the random variable $Z_{f}\left(G^{+}\right)$be the number of $k$-appropriate $W \subseteq M_{n}$ (i.e. $|W|=w=\left[1+k m_{s} \ln n\right]$ with $m$ from Definition 2.23) such that $(f, W)$ is bad. Let $\gamma$ denote the number of possible $k$-appropriate $W$. (The value of $\gamma$ is not used in the first stage of the argument.) Then, $E\left(Z_{f}\right)<\gamma n^{-|A|-1}$. So, by Markov's inequality,

$$
P_{n}\left(Z_{f} \geq \gamma / 2\right) \leq 2 E\left(Z_{f}\right) / \gamma<2 n^{-|A|-1} .
$$

But then, since there are only $n^{|A|}$ choices for $A$, a.a. for each $f$ at most half of the $W$ are bad for $f$.

Let $v$ denote $|B-A|=v(B / A)$. Each extension $g$ of $f$ to $B$ is contained in at most $\left(\begin{array}{l}n-v \\ w-v\end{array}\right) k$-appropriate $W$, since there are approximately $\left(\begin{array}{l}n-v \\ w-v\end{array}\right)$ choices for the elements which comprise $W-\operatorname{rng} g$. So at most $\left|N\left(f, A^{+}, B^{+}\right)\right|\left(\begin{array}{c}n-v \\ w-v\end{array}\right) k$-appropriate $W$ contain an extension of $f$ but at least $\gamma / 2$ do. Now note that $\gamma=\left(\begin{array}{l}n \\ w\end{array}\right)$.

Thus, a.a. for all $f$,

$$
\left|N\left(f, A^{+}, B^{+}\right)\right|\left(\begin{array}{c}
n-v \\
w-v
\end{array}\right) \geq \frac{1}{2}\left(\begin{array}{c}
n \\
w
\end{array}\right) .
$$

Noting that $\left(\begin{array}{l}n-v \\ w-v\end{array}\right)$ is approximately $\left(\begin{array}{l}n \\ w\end{array}\right)\left(\frac{w}{n}\right)^{v}$, we have

$$
\left|N\left(f, A^{+}, B^{+}\right)\right| \geq n^{-e\left(B^{+} / A^{+}\right)}(n / w)^{v} / 2 .
$$

Recalling that $w=\left[1+k m_{s} \ln n\right]$, this implies for every $f$, a.a.

$$
\left|N\left(f, A^{+}, B^{+}\right)\right|>n^{\delta\left(B^{+} / A^{+}\right)}(2 k s \ln n)^{-v}
$$

which establishes the lower bound $n^{\delta\left(B^{+} / A^{+}\right)}(\ln n)^{-c}$ by taking $c=v+1$.

2.26. Remark. The statement and proofs of of the probability analysis are based on the argument in [8]. The first author acknowledges discussions with Albert, Cherlin, Lachlan, and Laskowski on the details of the current argument, and supplemental remarks to the original paper by Spencer.

2.27. Remark. The irrationality hypothesis is necessary to make fruitful application of this result. If there exist $A \leq_{s} B$ with $\delta(B / A)=0$ then the lower bound we have established is less than one rather than tending to infinity as $n$ does. This destroys the argument of Theorem 2.30.

2.28. Remark. From Lemma 1.17 we have: Let $A^{+} \leq_{i} C^{+}$. There exists a $K$ such that a.a. for every embedding $f$ of $A^{+}$into an expansion $G^{+}$of $M_{n}$, there are fewer than $K L^{+}$-homomorphisms extending $f$ from $C^{+}$into $G^{+}$.

2.29. Proof of upper bound in Theorem 2.20. Since $B^{+}$is a primitive extension of $A^{+},(A b)^{+} \leq_{i} B^{+}$for any $b \in B-A$. Thus, by Remark 2.28 there are fewer than $K$ extensions $g_{i}$ with any fixed image of $b$. The range of each extension $g_{i}$ can intersect at most $K|B|^{2}$ other extensions so if $N\left(f, A^{+}, B^{+}\right)=s^{\prime}$, there is a set of $s=s^{\prime} /\left(K|B|^{2}+1\right)$ disjoint extensions. Let $p=|A|, v=v(B / A)=|B-A|$ and $e=e\left(B^{+} / A^{+}\right)$. For an appropriate constant $c<1$, there are less than $c n^{p}\left(n^{v s} / s !\right)$ pairs of a function $f$ taking $A$ into $M_{n}$ and a set of $s$ extensions (disjoint over $\operatorname{rng} f)\left\langle g_{1}, \ldots g_{s}\right\rangle$. The probability that each of the $g_{i}$ is an $L^{+}$-homomorphism 
is $n^{-e}$ so the probability of such a pair of a function and $s$ homomorphisms is at most $n^{p}\left(n^{v s} n^{-e s}\right) / s$ ! By Stirling's formula, this is less than $\frac{n^{p}\left(2.72 n^{v}\right)^{s} n^{-e s}}{(2 \pi)^{1 / 2} s^{s+1 / 2}(2.72)^{1 /(12 s+1)}}$ which is much less than 1 if $s \geq 3 n^{\delta\left(B^{+} / A^{+}\right)}$. (Observe that $n^{p}\left(\frac{2.72}{3}\right)^{s}$ tends to 0 as $n$ tends to infinity.) Thus, a.a. $s \leq 3 n^{\delta\left(B^{+} / A^{+}\right)}$. A.a. for each $f$,

$$
N\left(f, A^{+}, B^{+}\right)=s^{\prime} \leq 3\left(K|B|^{2}+1\right) n^{\delta\left(B^{+} / A^{+}\right)}
$$

proving Theorem 2.20.

We now want to show that each of the axioms for semigenericity has limit probability 1. Roughly, the program is to show that for $A^{+} \leq_{s} B^{+}$and $f: A \mapsto M_{n}$, the number of extensions of $f$ to 1-1 homomorphisms of $B^{+}$is much greater than the number of such extensions which fail to witness the definition of semigenericity. Since there are a bounded number of types of failure, it suffices to check each type separately as we do in the following argument.

In general, embeddings $f: A^{+} \mapsto M_{n}$ and $\hat{f}: B^{+} \mapsto M_{n}$ fail to witness semigenericity of $G^{+}$if

1. $\mathrm{cl}_{G^{+}}^{m}(\hat{f} B) \neq \hat{f} B \cup \mathrm{cl}_{G^{+}}^{m}(f A)$ or

2. $\operatorname{cl}_{G^{+}}^{m}(f A)$ and $\hat{f} B$ are not freely joined over $f A$ in $G^{+}$.

In considering $\phi_{A^{+}, B^{+}, C^{+}}^{m}$, we are fixing on $C^{+}$as a specific candidate for the isomorphism type of $\mathrm{cl}_{G^{+}}^{m}(f A)$.

2.30. Theorem. If $A^{+} \leq_{s} B^{+}$and $A^{+} \leq_{i} C^{+}$with $\left|C^{+}\right|<m$ then

$$
\lim _{n \rightarrow \infty} P_{n}\left(\phi_{A^{+}, B^{+}, C^{+}}^{m}\right)=1 \text {. }
$$

Proof. For any $f$ mapping $C$ into $M_{n}$, and a 1-1 homomorphism $f^{\prime}$ extending $f$ to $E,\left(G^{+}, f^{\prime}\right)$ fails as a witness for $C$ and $f$ if

i) $f^{\prime}$ is not an $L^{+}$-isomorphism or

ii) $\mathrm{cl}_{G^{+}}^{m}\left(f^{\prime} B\right) \neq f^{\prime} B \cup f C$ or

iii) $f C$ and $f^{\prime} B$ are not freely joined over $f A$ in $G^{+}$.

Note that $C^{+} \leq_{s} C^{+} \otimes_{A^{+}} B^{+}$and, letting $E^{+}$denote $C^{+} \otimes_{A^{+}} B^{+}, \delta\left(B^{+} / A^{+}\right)=$ $\delta\left(E^{+} / C^{+}\right)$. By Theorem 2.20, more specifically Paragraph 2.25, a.a. for each $f$,

$$
\left|N\left(f, C^{+}, E^{+}\right)\right|>n^{\delta\left(B^{+} / A^{+}\right)}(\log n)^{-c}
$$

where $c=v\left(B^{+} / A^{+}\right)+1$. For conditions i) and iii) consider any $F^{+}$which is an expansion of $E^{+}$by adding additional relations. Then $\delta\left(F^{+} / A^{+}\right)=v(E / A)-$ $e\left(F^{+} / A^{+}\right)$and $e\left(F^{+} / A^{+}\right)>e\left(E^{+} / A^{+}\right)$. By Theorem 2.20

$$
N\left(f, C^{+}, F^{+}\right)<c_{1} n^{\delta\left(F^{+} / A^{+}\right)}<n^{\delta\left(E^{+} / A^{+}\right)}(\log n)^{-c} .
$$

For condition ii) for any $D^{+} \in \mathcal{D}_{B^{+}, C^{+}}^{m}$,

$$
\delta\left(D^{+} B^{+} / C^{+}\right)<\delta\left(B^{+} C^{+} / C^{+}\right)=\delta\left(B^{+} / A^{+}\right) .
$$

If $C^{+}$is not strong in $D^{+} B^{+}$then by Lemma $2.28, N\left(f^{\prime}, C^{+}, D^{+} B^{+}\right)<K$. If $C^{+} \leq_{s} D^{+} B^{+}$then by Theorem 2.20, more specifically Paragraph 2.29, a.a.

$$
N\left(f, C^{+}, D^{+} B^{+}\right)<c_{1} n^{\delta\left(D^{+} B^{+} / C^{+}\right)}<n^{\delta\left(B^{+} C^{+} / C^{+}\right)}(\log n)^{-c} .
$$

Now the number of isomorphism types of extensions $C$ that have failures $f^{\prime}$ is bounded in terms of the cardinality of $A^{+}, B^{+}$, and $m$; it does not depend 
on $n$. If this number is $L$, the total number of failures of any sort is less than $L n^{\delta\left(E^{+} / A^{+}\right)}(\log n)^{-c}$. Thus, the probability that for each $f$, one of the extensions of $f$ witnesses $\phi_{A^{+}, B^{+}, C^{+}}^{m}$ tends to one as required.

2.31. Lemma. For every $m$, a.a. $\mathrm{cl}_{L^{+}}^{m}(\emptyset)=\emptyset$.

Proof. $A^{+} \subseteq \mathrm{cl}_{L^{+}}^{m}(\emptyset)$ just if $\delta\left(A^{+}\right)<0$. But in passing from $\mathbf{K}_{0}^{*}$ to $\mathbf{K}_{0}^{+}$(cf. Lemma 2.19), we have forbidden such $A$.

We collect our results in the following theorem which requires the definition of two theories.

2.32. Notation. Let $L$ contain only the equality symbol and let $L^{+}$be an arbitrary finite relational language containing $L$. Suppose probabilities are defined on finite $L^{+}$structures as in Definition 2.5 with the $\alpha_{i}$ and 1 linearly independent over the rationals. By $T^{\alpha}$, the almost sure theory of random $L^{+}$-structures we mean the collection of $L^{+}$-sentences which have limit probability 1 . Recall that $T_{\alpha}$ is the theory of the generic structures for $\mathbf{K}_{\alpha}$ (Definition 1.6) whose existence is guaranteed by Theorem 1.34 .

A theory $T$ has the finite model property if every theorem of $T$ has a finite model.

2.33. Theorem. Under the hypotheses in Notation 2.32, $T^{\alpha}$, the almost sure theory of random $L^{+}$-structures is the same as the theory $T_{\alpha}$ of the $\mathbf{K}_{\alpha}$-generic model. This theory is complete, stable, and nearly model complete. Moreover, it has the finite model property and has only infinite models so is not finitely axiomatizable.

Proof. By Theorem 2.30 and the choice of $\mathbf{K}_{0}^{+}$, every model of $T^{\alpha}$ is $\left(\mathbf{K}_{\alpha}, \leq_{\alpha}\right)$ semigeneric. By Corollary 1.31, $T^{\alpha}$ is nearly model complete. By Corollary 1.32 and Lemma 2.31, $T^{\alpha}$ is complete. Since the generic model for $\mathbf{K}_{\alpha}$ is semigeneric, $T^{\alpha}=T_{\alpha}$. [1] shows that $T_{\alpha}$ is stable.

Since each theorem of $T^{\alpha}$ has limit probability 1 , for arbitrarily large $n$, there is nonzero probability that there is a model of size $n$. Thus, $T^{\alpha}$ has the finite model property.

2.34. Remark. The major novelty of this result is the identification of the two theories, thereby obtaining the stability of $T^{\alpha}$ and the non-finite axiomatizability of $T_{\alpha}$. The notion of near model completeness specifies the precise degree of quantifier elimination in $T^{\alpha}$. In addition, we have extended the 0-1-law from a language with a single binary relation to an arbitrary finite relational language.

\section{REFERENCES}

[1] J.T. Baldwin and Niandong Shi. Stable generic structures. Annals of Pure and Applied Logic, 79: 1-35, 1996. CMP 96:13

[2] A. Baudisch. A new $\aleph_{1}$-categorical pure group. 1992.

[3] E. Hrushovski. A stable $\aleph_{0}$-categorical pseudoplane. preprint, 1988.

[4] D.W. Kueker and C. Laskowski. On generic structures. Notre Dame Journal of Formal Logic, 33:175-183, 1992. MR 93k:03032

[5] J. Lynch. Probabilities of sentences about very sparse random graphs. Random Structures and Algorithms, 3:33-53, 1992.

[6] S. Shelah. 0-1 laws. preprint 550, 199 ?

[7] S. Shelah. Zero-one laws with probability varying with decaying distance. Shelah 467, 199x. 
[8] S. Shelah and J. Spencer. Zero-one laws for sparse random graphs. Journal of A.M.S., 1:97115, 1988. MR 89i:05249

[9] F. Wagner. Relational structures and dimensions. In Automorphisms of first order structures, pages 153-181. Clarendon Press, Oxford, 1994. CMP 95:10

Department of Mathematics, Statistics and Computer Science, University of Illinois at Chicago, Chicago, Illinois 60607-7405

Department of Mathematics, Hebrew University of Jerusalem, Jerusalem, Israel 